\title{
Partial Response to Ceritinib in a Patient With Abdominal Inflammatory Myofibroblastic Tumor Carrying a TFG-ROS1 Fusion
}

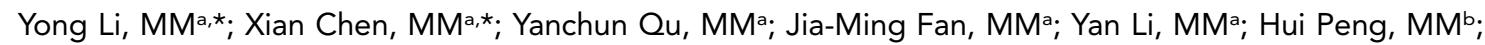
Yaojie Zheng, MMc; Yihong Zhang, MEc; and Hai-Bo Zhang, MDa

\section{ABSTRACT}

Inflammatory myofibroblastic tumor (IMT), a rare sarcoma, is primarily treated via resection of the mass. However, there is no standard treatment for recurrence or unresectable tumors. Almost $50 \%$ of IMTs carry ALK gene rearrangement that can be treated using ALK inhibitors, but therapeutic options for ALK-negative tumors are limited. This report describes a woman aged 22 years with unresectable ALK-negative IMT. Next-generation sequencing revealed a TFG-ROS1 fusion, and she had a partial response to the ROS1 inhibitor ceritinib. This report provides the first published demonstration of a patient with IMT with ROS1 fusion successfully treated using ceritinib. Our study suggests that targeting ROS1 fusions using the small molecule inhibitor shows promise as an effective therapy in patients with IMT carrying this genetic alteration, but this requires further investigation in large clinical trials.

J Natl Compr Canc Netw 2019;17(12):1459-1462 doi: $10.6004 /$ jnccn.2019.7360
aDepartment of Oncology, and bepartment of Pathology, Guangdong Provincial Hospital of Chinese Medicine, Guangzhou; and ' $\mathrm{OrigiMed,}$ Shanghai, China.
Inflammatory myofibroblastic tumor (IMT) is a rare soft tissue neoplasm containing myofibroblastic spindle cells with inflammatory cells. ${ }^{1}$ It usually arises in the lung, abdomen, or pelvis and affects primarily children and young adults. ${ }^{2}$ Symptoms of disease are often restricted to the involved organ, and $15 \%$ to $30 \%$ of patients present with fever, malaise, microcytic anemia, weight loss, thrombocytosis, elevated erythrocyte sedimentation rate, or polyclonal hypergammaglobulinemia. ${ }^{3}$

Surgery is the primary management for patients with localized IMT; however, there is no standard treatment for patients with advanced and/or unresectable IMT. At the molecular level, gene fusion is a feature of IMT and also a common molecular feature of soft tissue sarcoma. Almost half of IMT cases carry $A L K$ gene rearrangement that can be treated using $A L K$ inhibitors, ${ }^{4-6}$ but therapeutic options for $A L K$-negative tumors are limited. $A L K$-negative IMTs may be more aggressive, with a higher frequency of metastasis compared with $A L K$-positive IMTs. ${ }^{7}$ Recent studies have described novel fusions involving ROS1, NTRK, $R E T$, and PDGFR $\beta$ genes in a subset of $A L K$-negative cases. $^{8-10}$ Little is known on the genomic level about potential oncogenic drivers in this subset of IMTs. Given the rarity of IMT, few prospective studies focusing on its targeted therapy are available. This report presents a case of TFG-ROS1-positive IMT that developed in the abdominal cavity and shrank remarkably after treatment with ceritinib.

\section{Case Description}

On September 3, 2018, a woman aged 22 years with no relevant past medical history presented to a local hospital with multiple masses in the left abdominal cavity and retroperitoneum seen on CT scan, and polypoid hyperplasia in the large intestine seen on colonoscopy. PET scan showed enhanced FDG-avid uptake in the retroperitoneum, suggesting a malignant tumor in the abdomen. The patient was subsequently referred to our hospital, where she underwent gastrojejunostomy on October 8, 2018. Postoperative pathologic diagnosis was 
intermediate spindle cell tumor of omental nodules. Under a light microscope, the tumor was found to be composed of proliferating fusiform fibroblasts/myofibroblasts and collagen fibers; fibroblasts were displayed with the shapes of striate, star, and bundle; and the mesenchyme contained abundant lymphocytes and plasmocytes. Immunohistochemical (IHC) staining of CD34, SMA, Ki67, $\beta$-catenin, CR, IgG4, CD38, CK, EMA, calretinin, and desmin were positive, whereas $A L K, D O G 1, C D 117$, S100, CD30, HMB45, Melan-A, STAT6, IgG, Era, progesterone receptor, and caldesmon were negative. Based on her IHC results and the tumor characteristics seen on light microscopy, the patient was finally diagnosed with abdominal IMT.

On November 7, 2018, the patient was treated with cisplatin $(40 \mathrm{mg} / \mathrm{d}$ intravenously) and methotrexate ( $25 \mathrm{mg} / \mathrm{d}$ intravenously) as adjuvant chemotherapy for 1 cycle. Meanwhile, next-generation sequencing (NGS) of the tumor tissue was performed at OrigiMed. A TFGROS 1 fusion involving exons 1-4 of TFG and exons 35-43 of ROS1 was identified (Figure 1). The fusion retained the intact kinase domain of ROS1. During reexamination in December 2018, PET/CT scan (Figure 2A, B) showed enhanced hypermetabolic lesions in the retroperitoneum and mesenteric roots and the left lower abdomen. The patient withdrew from chemotherapy because of severe adverse effects and started ceritinib $(450 \mathrm{mg} / \mathrm{d})$ monotherapy on December 8, 2018. After 2.5 months of ceritinib treatment, PET/CT scan showed remarkable shrinkage and even disappearance of masses in the retroperitoneum and mesenteric roots and the left lower abdomen (Figure 2C, D). Clinical efficacy was evaluated as partial response. The patient developed vomiting and diarrhea after ceritinib treatment. These adverse effects disappeared after a ceritinib dose reduction to $300 \mathrm{mg} / \mathrm{d}$ beginning in June 2019. In August 2019, she restarted ceritinib at $450 \mathrm{mg} / \mathrm{d}$, and no adverse effects occurred.

\section{Discussion}

According to WHO classification, IMT is currently a distinctive neoplasm composed of myofibroblasts accompanied by an inflammatory infiltrate of lymphocytes,

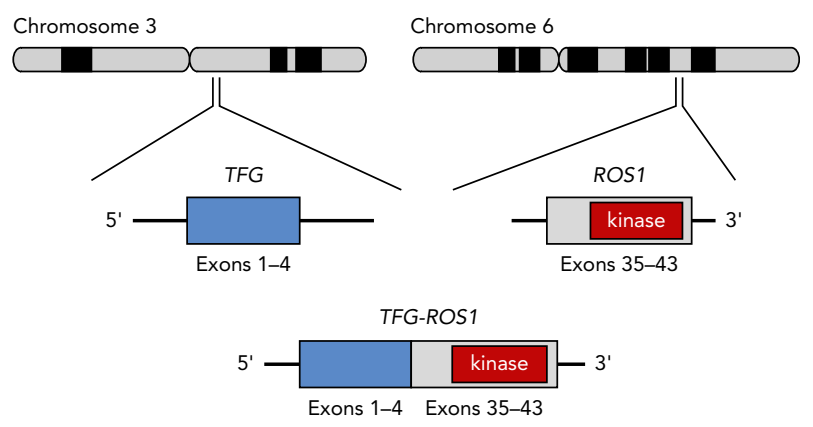

Figure 1. Genomic fusion of exons 1-4 of TGF to exons 35-43 of ROS1. plasma cells, and/or eosinophils. ${ }^{11}$ Surgery is considered the main therapeutic approach for IMT, because other treatment approaches for patients with advanced and/or unresectable IMT are extremely limited. Importantly, IMT has drawn increasing attention because of its molecular characteristics and actionable targets reported in recent years. This report describes a woman aged 22 years who was diagnosed with $A L K$-negative abdominal IMT based on IHC results and tumor characteristics seen on light microscopy, and in whom NGS testing identified a TFG-ROS1 fusion. The patient achieved partial response after treatment with ceritinib. This report provides a new potential therapeutic option for some patients with ROS1 gene rearrangement of this tumor type.

ROS1 is a receptor tyrosine kinase involved in the regulation of several cellular processes, including proliferation, differentiation, apoptosis, and cell migration. ${ }^{12}$ Substantial evidence supports ROS1 as a key player in many solid tumors, including non-small cell lung cancer (NSCLC), cholangiocarcinoma, gastric adenocarcinoma, and colorectal cancer. ${ }^{13}$ ROS1 rearrangements create fusion proteins that result in constitutive activation of the tyrosine kinase and acceleration of cellular proliferation. ${ }^{14,15}$ ROS1 gene fusions were recently identified in a subset of $A L K$ negative IMT, ${ }^{8,16}$ suggesting a new diagnostic marker for this group of neoplasms. Thus far, only 2 ROS1 fusion partners, TFG and YWHAE, have been identified in IMTs, with TFG the most common partner., ${ }^{8,16,17}$ In our patient's case, because complete tumor resection was not possible, tumor biopsies were subjected to NGS testing and a TFGROS1 fusion was identified. The chromosomal rearrangement was identified between regions $3 \mathrm{p} 12$ and $6 \mathrm{q} 22$, causing fusion between exon 4 of TFG and exon 35 of ROS1. This result is consistent with previous reports on TFG-ROS1 fusion, the breakpoints of which are located between exon 4 of TFG and exon 34 or 35 of ROS1, leading to constitutive activation of $R O S 1$ that contains its intact kinase domain.8,18

The discovery of ROS1 fusions in patients with IMT has facilitated the clinical development of ROS1 inhibitors, including crizotinib and ceritinib. Crizotinib is a small molecule tyrosine kinase inhibitor (TKI) that was found to actively inhibit $A L K, M E T$, and ROS1. Crizotinib was originally developed as an anti-MET molecule, ${ }^{19}$ but subsequently showed significant activity in $A L K$-driven tumors and was FDA-approved for $A L K$-positive advanced NSCLC. ${ }^{20}$ Crizotinib has also shown remarkable efficacy against ROS1-positive lung cancer and was the first TKI approved for ROS1-positive advanced NSCLC. ${ }^{21}$ A patient aged 14 years with pulmonary IMT with a TFG-ROS1 fusion achieved continuous remission ${ }^{22}$ and a boy aged 8 years with treatment-refractory $A L K$-negative IMT harboring a TFG-ROS1 fusion experienced a dramatic response after treatment with crizotinib. ${ }^{8}$ However, another study found that a patient 
A

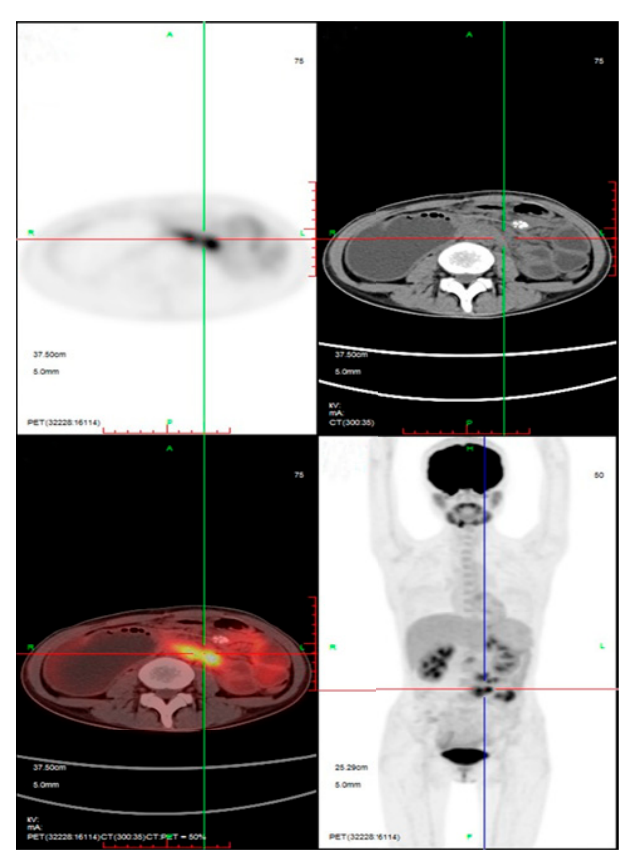

C

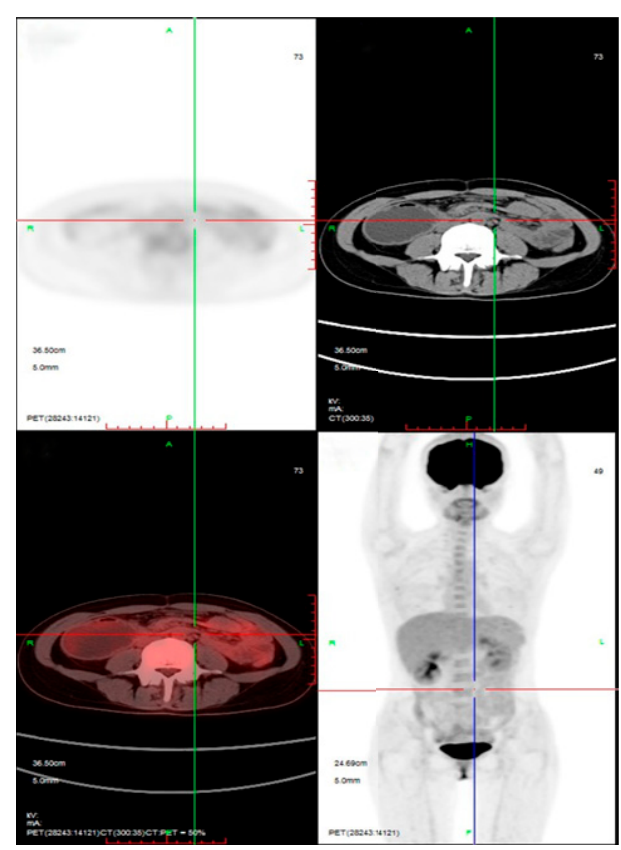

B

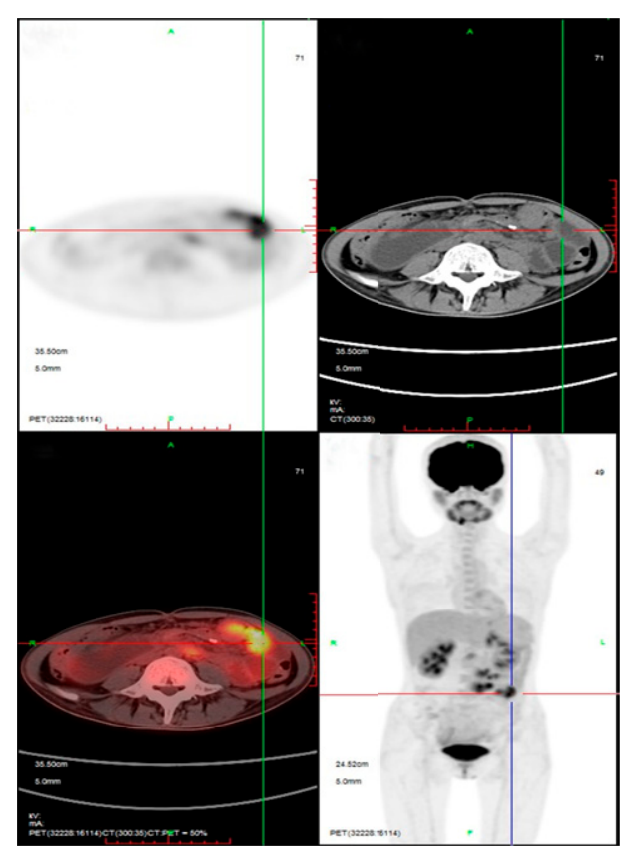

D

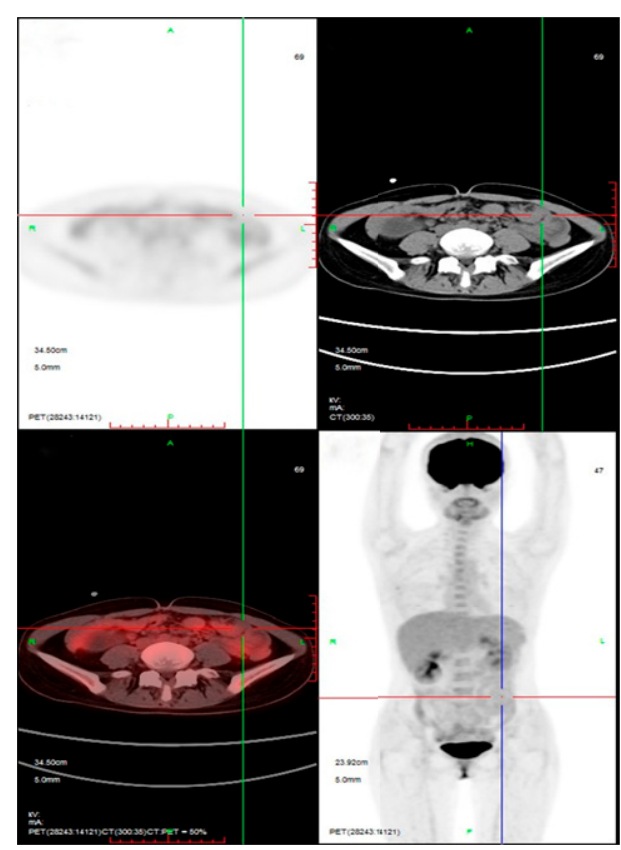

Figure 2. PET/CT imaging showing enhanced hypermetabolic lesions in the (A) retroperitoneum and mesenteric roots and (B) left lower abdomen before ceritinib treatment, and remarkable shrinkage and even disappearance of masses in the (C) retroperitoneum and mesenteric roots and (D) left lower abdomen after 2.5 months of ceritinib treatment.

aged 59 years with metastatic gastric IMT carrying a TFG-ROS1 fusion and treated with crizotinib showed a poor response. ${ }^{23}$

Ceritinib, a selective oral TKI of $A L K$, is similar in action to crizotinib without $M E T$-inhibiting ability. It was FDA-approved for treatment of crizotinib-resistant $A L K$-positive NSCLC. In enzymatic assays, ceritinib showed 20 times greater potency than crizotinib against $A L K{ }^{24}$ In addition, ceritinib has shown comparable activity to crizotinib against $R O S 1 .{ }^{25,26}$ In an open-label multicenter phase II study, ceritinib showed potent clinical activity in patients with NSCLC harboring ROS1 rearrangement, with a $62 \%$ objective response rate and 9.3 and 19.3 months of median progression-free survival among all patients and those who were crizotinib-naïve, respectively. ${ }^{27}$ Ceritinib has 
also shown clinical activity in patients with IMT. A male patient aged 32 years with an $A L K$-rearranged IMT showed partial response to ceritinib after failure of crizotinib, ${ }^{28}$ and another patient with a highly aggressive $A L K$-rearranged IMT arising in the pleural cavity showed a drastic response to $2 A L K$ inhibitors: ASP3026 and ceritinib. ${ }^{5}$ In our study, the patient began ceritinib therapy and achieved partial response with an excellent tolerance after 2.5 months. This is the first report of a patient with ROS1-rearranged IMT being treated successfully with ceritinib.

\section{Conclusions}

Because no standard treatment exists for patients with advanced and/or unresectable IMT, we hope that the identification of actionable genetic alterations will redefine management strategies for this disease. We identified a TFG-ROS1 fusion in a patient with IMT, who showed remarkable tumor shrinkage and achieved partial response after treatment with ceritinib. Our study provides the first report of a patient with ROS1rearranged IMT being treated successfully with ceritinib. However, the response seen in this patient, although promising, requires further investigation in large clinical trials.

Submitted for publication June 24, 2019; accepted for publication September 13, 2019.

Disclosures: Drs. Zheng and Y. Zhang have disclosed that they are employees of OrigiMed. The remaining authors have disclosed that they have not received any financial consideration from any person or organization to support the preparation, analysis, results, or discussion of this article.

Correspondence: Hai-Bo Zhang, MD, Department of Oncology, Guangdong Provincial Hospital of Chinese Medicine, Guangzhou 510120, China. Email: haibozh@aliyun.com

\section{References}

1. Su LD, Atayde-Perez A, Sheldon S, et al. Inflammatory myofibroblastic tumor: cytogenetic evidence supporting clonal origin. Mod Pathol 1998; 11:364-368.

2. Gleason BC, Hornick JL. Inflammatory myofibroblastic tumours: where are we now? J Clin Pathol 2008;61:428-437.

3. Coffin CM, Watterson J, Priest JR, et al. Extrapulmonary inflammatory myofibroblastic tumor (inflammatory pseudotumor). A clinicopathologic and immunohistochemical study of 84 cases. Am J Surg Pathol 1995;19:859-872.

4. Coffin CM, Patel A, Perkins S, et al. ALK1 and p80 expression and chromosomal rearrangements involving 2 p23 in inflammatory myofibroblastic tumor. Mod Pathol 2001;14:569-576.

5. Ono A, Murakami H, Serizawa $M$, et al. Drastic initial response and subsequent response to two ALK inhibitors in a patient with a highly aggressive ALK-rearranged inflammatory myofibroblastic tumor arising in the pleural cavity. Lung Cancer 2016;99:151-154.

6. Rao N, Iwenofu H, Tang B, et al. Inflammatory myofibroblastic tumor driven by novel NUMA1-ALK fusion responds to ALK inhibition. J Natl Compr Canc Netw 2018;16:115-121.

7. Coffin $\mathrm{CM}$, Hornick JL, Fletcher CD. Inflammatory myofibroblastic tumor: comparison of clinicopathologic, histologic, and immunohistochemical features including ALK expression in atypical and aggressive cases. Am J Surg Pathol 2007;31:509-520.

8. Lovly CM, Gupta A, Lipson D, et al. Inflammatory myofibroblastic tumors harbor multiple potentially actionable kinase fusions. Cancer Discov 2014;4:889-895.

9. Takahashi A, Kurosawa M, Uemura M, et al. Anaplastic lymphoma kinasenegative uterine inflammatory myofibroblastic tumor containing the ETV6-NTRK3 fusion gene: a case report. J Int Med Res 2018;46: 3498-3503.

10. Antonescu CR, Suurmeijer AJ, Zhang L, et al. Molecular characterization of inflammatory myofibroblastic tumors with frequent $A L K$ and ROS1 gene fusions and rare novel RET rearrangement. Am J Surg Pathol 2015; 39:957-967.

11. Jo $W Y$, Fletcher CD. WHO classification of soft tissue tumours: an update based on the 2013 (4th) edition. Pathology 2014;46:95-104.

12. Robinson DR, Wu YM, Lin SF. The protein tyrosine kinase family of the human genome. Oncogene 2000;19:5548-5557.

13. Davies KD, Doebele RC. Molecular pathways: ROS1 fusion proteins in cancer. Clin Cancer Res 2013;19:4040-4045.

14. Takeuchi K, Soda M, Togashi Y, et al. RET, ROS1 and ALK fusions in lung cancer. Nat Med 2012;18:378-381.
15. Charest A, Kheifets V, Park J, et al. Oncogenic targeting of an activated tyrosine kinase to the Golgi apparatus in a glioblastoma. Proc Natl Acad Sci USA 2003;100:916-921.

16. Chang JC, Zhang L, Drilon AE, et al. Expanding the molecular characterization of thoracic inflammatory myofibroblastic tumors beyond ALK gene rearrangements. J Thorac Oncol 2019;14:825-834.

17. Hornick JL, Sholl LM, Dal Cin P, et al. Expression of ROS1 predicts ROS1 gene rearrangement in inflammatory myofibroblastic tumors. Mod Pathol 2015;28:732-739.

18. Rossing M, Yde CW, Sehested A, et al. Genomic diagnostics leading to the identification of a TFG-ROS1 fusion in a child with possible atypical meningioma. Cancer Genet 2017;212-213:32-37.

19. Zou HY, Li Q, Lee JH, et al. An orally available small-molecule inhibitor of c-Met, PF-2341066, exhibits cytoreductive antitumor efficacy through antiproliferative and antiangiogenic mechanisms. Cancer Res 2007;67:4408-4417.

20. Kwak EL, Bang YJ, Camidge DR, et al. Anaplastic lymphoma kinase inhibition in non-small-cell lung cancer. N Engl J Med 2010;363:1693-1703.

21. Roskoski R Jr. ROS1 protein-tyrosine kinase inhibitors in the treatment of ROS1 fusion protein-driven non-small cell lung cancers. Pharmacol Res 2017;121:202-212.

22. Mai S, Xiong G, Diao D, et al. Case report: crizotinib is effective in a patient with ROS1-rearranged pulmonary inflammatory myofibroblastic tumor. Lung Cancer 2019;128:101-104.

23. Daniel S, Gay LM, Vergilio JA, et al. A clinical and genomic profile of inflammatory myofibroblastic tumors [abstract]. J Clin Oncol 2017; 35(Suppl 15):Abstract 1538.

24. Santarpia M, Daffinà MG, D'Aveni $A$, et al. Spotlight on ceritinib in the treatment of ALK+ NSCLC: design, development and place in therapy. Drug Des Devel Ther 2017;11:2047-2063.

25. Shaw AT, Engelman JA. Ceritinib in ALK-rearranged non-small-cell lung cancer. N Engl J Med 2014;370:1189-1197.

26. Facchinetti $F$, Loriot $Y$, Kuo MS, et al. Crizotinib-resistant ROS1 mutations reveal a predictive kinase inhibitor sensitivity model for ROS1- and ALK-rearranged lung cancers. Clin Cancer Res 2016;22:5983-5991.

27. Lim SM, Kim HR, Lee JS, et al. Open-label, multicenter, phase II study of ceritinib in patients with non-small-cell lung cancer harboring ROS1 rearrangement. J Clin Oncol 2017;35:2613-2618.

28. Mansfield AS, Murphy SJ, Harris FR, et al. Chromoplectic TPM3-ALK rearrangement in a patient with inflammatory myofibroblastic tumor who responded to ceritinib after progression on crizotinib. Ann Oncol 2016; 27:2111-2117 